\title{
Variation de l'énergie métabolisable du pois d'Angole (Cajanus cajan) cru et cuit chez le poulet et la pintade
}

\author{
C. Chrysostome ${ }^{1} \mathrm{Xu}$ Bing An ${ }^{2}$ M. Bonou ${ }^{3}$ \\ P. Delpech ${ }^{4}$
}

\section{Mots-clés}

Cajanus cajan - Poulet - Pintade Valeur énergétique.

\begin{abstract}
Résumé
Des graines de pois d'Angole (Cajanus cajan) crues et autoclavées ont été étudiées par substitution à 40 p. 100 dans un régime à base de maïs. La composition chimique de ces graines et leur concentration en énergie métabolisable apparente ont été obtenues par une technique de gavage semihumide chez le poulet en croissance et la pintade adulte. Les valeurs obtenues pour le maïs et le pois d'Angole cuit étaient identiques pour les deux espèces aviaires (3 $220 \mathrm{kcal}$ ). L'autoclavage a significativement amélioré l'énergie métabolisable du pois d'Angole mais différemment suivant l'espèce (2 $167 \mathrm{kcal}$ pour le poulet, $870 \mathrm{kcal}$ pour la pintade). La pintade gavée a mal digéré la graine crue de sorte que la méthode de mesure de la concentration énergétique ne convient pas pour cette espèce.
\end{abstract}

\section{INTRODUCTION}

La légumineuse Cajanus cajan L, appelée communément pois d'Angole, est très répandue dans la zone intertropicale des trois continents (3). En Afrique occidentale cette plante est surtout consommée sous forme de fourrage. La graine peut constituer une source non conventionnelle de protéines. Nwokolo et Oji ont mesuré au Nigeria la valeur énergétique chez le poulet de deux variétés, l'une brune et l'autre blanche, en l'état et après autoclavage (9). Ce traitement améliore la valeur énergétique de 7 p. 100 en moyenne. Les auteurs ont utilisé la méthode traditionnelle de mesure de l'énergie métabolisable apparente (EMA) avec une alimentation à volonté et en substituant le pois d'Angole au tourteau de soja. Cette étude a été reprise ici en comparant les valeurs obtenues chez le poulet et la pintade et en utilisant la technique de gavage selon le protocole de Lessire (6). Cette méthode a semblé mieux adaptée d'une part à la mesure de produit non conventionnel inappétant et d'autre part aux pintades qui ont tendance au gaspillage. Selon Mohamed et coll., la même difficulté est rencontrée chez le canard (8). Il a semblé utile par ailleurs de vérifier que le nombre de gavages n'affectait pas la digestion ou le métabolisme des deux espèces.

1. Université nationale du Bénin, Département de production animale, Faculté des sciences agronomiques, BP 526, Cotonou, Bénin

2. Institut des sciences et technologies, Région de Hengshui 76, Xin Hau, BP 053000, Rép. pop. Chine

3. Institut de développement rural, Ouagadougou, Burkina Faso

4. Institut national agronomique, Chaire de zootechnie, Centre de Paris-Grignon, 78850 Thiverval-Grignon

\section{MATERIEL ET METHODES}

Neuf poulets mâles du croisement ISA 757 âgés de 16 semaines et neuf pintades mâles de réforme de souche Galor ont été utilisés. Ils ont été élevés individuellement dans des cages à métabolisme. Entre chaque période expérimentale ils ont reçu un aliment complet et équilibré du commerce pour poulettes en croissance. Ils ont été abreuvés individuellement à volonté. Durant les périodes de mesure ils ont reçu l'un des trois régimes expérimentaux suivants : - aliment $\mathrm{m}$, formé de maïs exclusivement ;

- aliment cr, formé de 60 p. 100 de maïs et de 40 p. 100 de graines crues de pois d'Angole ;

- aliment ct, formé de 60 p. 100 de maïs et de 40 p. 100 de graines cuites de pois d'Angole.

Les graines de pois d'Angole de variété brune provenaient du domaine expérimental de l’Université nationale du Bénin.

Tous les aliments étaient en farine. La cuisson des graines de pois d'Angole a été effectuée dans un autocuiseur pendant $2 \mathrm{~h}$ (1 kg de graines pour $2 \mathrm{~kg}$ d'eau). Les graines ont ensuite été séchées pendant $24 \mathrm{~h}$ dans une étuve à $65^{\circ} \mathrm{C}$. Avec le régime au maïs et celui contenant le pois d'Angole cuit, deux rythmes de gavage ont été réalisés. La quantité journalière distribuée en une ou deux fois était identique, indicée 1 et 2 dans la désignation des lots. Les distributions ont été séparées de huit heures de manière à permettre une vidange de la partie supérieure du tractus digestif.

Pour chaque espèce, cinq lots ont ainsi été constitués $\mathrm{m}_{1}$ et $\mathrm{m}_{2}$ pour le maïs ; cr pour le pois d'Angole cru ; $\mathrm{ct}_{1}$ et ct $\mathrm{ct}_{2}$ pour le pois d'Angole cuit. Tous les animaux ont reçu successivement les cinq traitements avec une période de récupération de 48 h entre deux périodes de mesure. 
La technique de gavage semi-humide (mélange à poids égal d'aliment et d'eau) a été réalisée à l'aide d'une pompe à graisse manuelle modifiée permettant de pousser l'aliment dans l'œsophage jusqu'au jabot (12). La quantité ingérée a été mesurée en pesant la pompe avant et après gavage et en mesurant la matière sèche du mélange.

Les excrétats totaux ont été collectés pendant les deux jours qui ont suivi le gavage. Les fientes ont été lyophilisées.

L'énergie des aliments et des excrétats a été déterminée à la bombe calorimétrique adiabatique sur un échantillon de $2 \mathrm{~g}$ environ. L'énergie métabolisable apparente a été calculée suivant l'équation de substitution de Potter (10). L'EMA corrigée pour un bilan azoté nul $\left(\mathrm{EMA}_{\mathrm{n}}\right)$ a été obtenue à partir de l'équation indiquée par Larbier et Leclercq (4). Les écarts-types à la moyenne ont été calculés selon la méthode proposée par Yoshida (13).

Les données ont été soumises à une analyse de variance. L'aliment et l'espèce étaient les effets testés à l'aide de la comparaison des moyennes selon Newman-Keuls avec un risque de 5 p. 100.

\section{RESU LTATS ET DISCU SSIO N}

Dans le tableau I sont rassemblés les résultats de l'analyse bromatologique et la concentration en énergie brute des graines de pois d'Angole crues et cuites. Les différences constatées entre les deux états sont faibles mais concernent toutes les caractéristiques analytiques. Les valeurs de cette étude sont proches de celles observées par Nwokolo et Oji (9) quoique la teneur en protéines soit plus faible (21 à 22 p. 100) et la teneur en lipides plus élevée (3 p. 100). León et coll. au Venezuela observent de plus faibles teneurs en protéines (20 p. 100) et en lipides (1 p. 100) (5). Ces variations peuvent résulter des variétés, des modalités de culture et des conditions de récolte et de stockage.

Par rapport aux résultats nigérians, la plus faible concentration en énergie brute (EB) des graines crues peut s'expliquer par une teneur plus faible en protéines et surtout en lipides, et par une concentration plus élevée en minéraux. L'autoclavage a probablement solubilisé une partie des minéraux et peut-être des glucides. Il a pu endommager les parois végétales, ce qui expliquerait la diminution de la cellulose brute.

L'énergie métabolisable apparente corrigée ne différait pas de la valeur non corrigée car le bilan azoté est demeuré inférieur à $1 \mathrm{~g}$ et le poids des animaux n'a pratiquement pas changé entre le début et la fin de l'expérience. Le gavage en une ou deux fois n'a pas influencé le résultat obtenu (tableau II).

L'EMA du pois d'Angole cru était plus faible que celle obtenue par León et coll. (5) et par Nwokolo et Oji (9). Mais la composition des graines différait également (tableau I). Chez la pintade une valeur très inférieure à $1000 \mathrm{kcal}$, significativement différente de celle obtenue pour le poulet $(\mathrm{p}<0,01)$, a été observée. Il semble exister une perturbation des processus digestifs qui est illustrée par les variations du rapport EMA/EB pour les différents aliments testés. Elle pourrait être due à la méthode d'alimentation utilisée. Le gavage nécessite l'emploi d'aliments qui ne sont pas susceptibles de modifier le transit digestif sous peine d'obtenir des valeurs inexploitables, comme Auvergne le constate chez les canards gavés en vue de la production de foie gras (1).

La cuisson améliore l'EMA de 7 p. 100 chez le poulet, comme l'ont observé les auteurs nigérians (tableau II). Nwokolo et Oji obtiennent des résultats nettement supérieurs (3 $130 \mathrm{kcal}$ ) (9), mais cela résulte probablement de l'emploi du tourteau de soja au lieu du maïs pour la méthode de substitution. L'autoclavage semble donc avoir détruit au moins une partie des facteurs anti-nutritionnels qu'ils contenaient, car la plupart d'entre eux sont thermolabiles, selon Liener (7). Ce serait le cas des inhibiteurs de trypsine trouvés par Brochers et Ackerson (2).

Les valeurs énergétiques du maïs et du pois d'Angole cuit sont équivalentes pour les deux espèces (tableau II).

A partir de l'analyse bromatologique des graines de pois d'Angole crues et cuites, une estimation de la concentration en EMA a été calculée suivant les équations de prédiction de Sibbald et de Carré et Brillouet, citées par Larbier et Leclercq (4). Les valeurs obtenues sont rapportées dans le tableau I. L'équation de Carré et Brillouet paraît plus exacte car elle tient compte de l'EB. Toutefois ces équations sont destinées à être appliquées à des aliments composés et non à des MP, et leur emploi dans ces conditions est donc indicatif.

\section{- CON CLUSION}

Les graines de pois d'Angole doivent être cuites avant leur incorporation dans les régimes pour volailles et particulièrement pour les pintades. De nouvelles déterminations de l'EMA demeurent nécessaires avec différentes techniques plus adaptées aux pintades, d'une part, et à la faible appétance du pois d'Angole, d'autre part.

Tableau I

Composition chimique de graines de pois d'Angole crues ou cuites et calcul de l'énergie métabolisable

\section{Composition}

Matière sèche (MS) (\% produit)

Protéines brutes (PB) (\% MS)

Matières grasses (MG) (\% MS)

Cellulose brute (CB) (\% MS)

Matières minérales (Ce) (\% MS)

Energie brute mesurée (kcal/MS)

EMA calculée (kcal) (Sibbald, 1980) ${ }^{1}$

EM A calculée (kcal) (Carré et Brillouet, 1989) ${ }^{2}$

1. $\mathrm{EMA}_{\mathrm{n}}=3951+54,4 \mathrm{MG}-88,7 \mathrm{CB}-40,8 \mathrm{Ce}$ (kcal)

2. $\mathrm{EMA}_{\mathrm{n}}=0,913 \mathrm{~EB}-18,5 \mathrm{~PB}-109,5 \mathrm{CB}$ (kcal)

\section{Pois d'Angole cru 4}

89,3

23,5

1,5

11,8

5,8

3893

2754

1816
Pois d'Angole cuit 5

91,8

24,3

2,2

9,6

4,2

4183

3051

2306 
Tableau II

Valeurs d'énergie métabolisable de la graine de pois d'Angole crue ou cuite mesurées sur des pintades adultes et des poulets en fin de croissance

\begin{tabular}{|c|c|c|c|c|c|}
\hline \multirow[b]{2}{*}{ Aliments testés } & \multicolumn{2}{|c|}{ Poulet } & \multicolumn{2}{|c|}{ Pintade } & \multirow[b]{2}{*}{$\begin{array}{c}\text { Effet } \\
\text { espèce animale }\end{array}$} \\
\hline & $\begin{array}{c}\mathrm{EMA}_{\mathrm{n}} \\
\mathrm{kcal} / \mathrm{kg} \mathrm{de} \mathrm{MS}\end{array}$ & $\begin{array}{c}\text { EMA/EB } \\
\%\end{array}$ & $\begin{array}{c}\text { EMA }_{n} \\
\mathrm{kcal} / \mathrm{kg} \text { de MS }\end{array}$ & $\begin{array}{c}\text { EMA/EB } \\
\%\end{array}$ & \\
\hline M aïs $\left(m_{1}\right)$ & $3236^{a} \pm 82$ & 80,7 & $3169^{a} \pm 89$ & 79,0 & NS \\
\hline M aïs $\left(m_{2}\right)$ & $3293^{a} \pm 43$ & 82,1 & $3180^{a} \pm 108$ & 79,3 & NS \\
\hline Pois d'Angole cru (cr) & $2167^{c} \pm 44$ & 55,7 & $870^{c} \pm 92$ & 22,3 & $p<0,001$ \\
\hline Pois d'Angole cuit ( $\mathrm{ct}_{1}$ ) & $2539 b \pm 34$ & 60,7 & $2533^{b} \pm 75$ & 60,6 & NS \\
\hline Pois d'Angole cuit ( $\left.\mathrm{ct}_{2}\right)$ & $2459 b \pm 59$ & 58,9 & $2538^{b} \pm 44$ & 60,7 & NS \\
\hline
\end{tabular}

Dans une même colonne, les valeurs suivies de la même lettre ne sont pas significativement différentes $(\mathrm{p}<0,05)$

\section{Remerciements}

Les auteurs remercient la Station de recherches avicoles, INRA de Tours-Nouzilly, pour la lyophilisation des échantillons ainsi que J.L. Le Pierres et A. Crispin pour leur aide technique.

\section{BIBLIO G RAPHIE}

1. AUVERGNE A., 1992. Facteurs de variation de la composition corporelle et tissulaire des canards avant et après gavage. Thèse doct. Etat, Institut national polytechnique, Toulouse, France, p. 252.

2. BROCHERS R., ACKERSON C.W., 1950. The nutritive value of legume seeds. X. Effect of autoclaving and the trypsin inhibitor test for 17 species. J. N utr., 41: 339.

3. JOHNSON R.M., RAYMOND W.D., 1964. The chemical composition of some tropical food plants: II. Pigeon peas and cow peas. J. trop. Prod. Inst., 6: 68-73.

4. LARBIER M., LECLERCQ B., 1992. Nutrition et alimentation des volailles. Paris, France, INRA Editions, $355 \mathrm{p}$.

5. LEON A., ANGULO I., JARAM ILLO M., REQUENA F., CALABRESSE H., 1992. Composición química y valor nuticional de granos de leguminosas tropicales. In: $4^{\mathrm{e}}$ Congreso de Avicultura nacional, Carabelleda, Caracas, Venezuela, 6-8 mayo 1992, 106 p.

\section{Summary}

Chrysostome C., Xu Bing An, Bonou M., Delpech P. Variations in the metabolizable energy of raw and autoclaved pigeon pea (Cajanus cajan) in chickens and guinea fow 1

Raw and autoclaved pigeon pea (Cajanus cajan) seeds, substituted at $40 \%$ from corn, were studied. The chemical composition and apparent metabolizable energy (AME) of the seeds were obtained in growing chickens and adult guinea fowl using a semi-wet force feeding technique. The values obtained were similar in both species $(3200 \mathrm{kcal})$. The autoclave treatment significantly, but differently, improved AME depending on the species $(2167 \mathrm{kcal}$ in chicks, $870 \mathrm{kcal}$ in guinea fowl). Force fed guinea fowl did not digest raw seeds well. Therefore, this AME measuring method is not adequate for this species.

Key words: Cajanus cajan - Chicken - Guinea fowl - Energy value.
6. LESSIRE M., 1990. Effect of feeding technique ad libitum dry or force feeding on the metabolizable energy of raw materials for poultry. Br. Poult. Sci., 31: 783-793.

7. LIENER I.E., 1966. Toxic substances associated with seed proteins. In: World protein ressources. Adv. Chem. Ser., 57: 178

8. MOHAMED K., LECLERCQ B., ANWWAR A., EL-ALAILY H., SO LIMAN H., 1984. A comparative study of metabolizable energy in duckling and domestic chicks. Anim. Feed Sci. Technol., 11: 199-210.

9. NWOKOLO E., OJI U.I., 1985. Variation in metabolizable energy of raw or autoclaved white and brown varieties of three tropical grain legumes. Anim. Feed Sci. Technol., 13: 141-146.

10. POTTER L.M., 1971. The precision of measuring metabolizable energy in poultry feedstuffs. In: Proc. 32nd Annu. Meet. Anim. Nutr. Res. Council, Washington, DC, USA, p. 6-11.

11. SCOTT M.L., NESHEIM M.C., YOUNG R.J., 1976. Nutrition of the chicken, 2nd ed. Ithaca, NY, USA, Scott and Associates, $555 \mathrm{p}$.

12. TEETER R.G., SMITH M.O., MURRAY E., 1984. Force feeding methodology and equipment for poultry. Poult. Sci., 63: 573-575.

13. YOSHIDA H., 1972. Evaluation of error of variance of metabolizable energy of feed ingredient. Jpn. Poult. Sci., 9: 281-285.

Reçu le 27.1.95, accepté le 22.6.98

\section{Resumen}

Chrysostome C., Xu Bing An, Bonou M., Delpech P. Variación de la energía metabolizable del guisante de Angola (Cajanus cajan) crudo y cocido en el pollo y la pintada

Se estudiaron granos de guisante de Angola (Cajanus cajan) crudos y autoclavados para la substitución de $40 \%$ de un régimen a base de maíz. La composición química de estos granos y su concentración en energía metabolizable aparente se obtuvo mediante una técnica de atracón semi húmedo en el pollo en crecimiento y la pintada adulta. Los valores obtenidos para el maíz y el guisante de Angola cocido fueron idénticos para las dos especies de aves (3 $220 \mathrm{kcal}$ ). El autoclavado mejoró significativamente la energía metabolizable del guisante de Angola, pero difirió según la especie (2 $167 \mathrm{kcal}$ para el pollo, $870 \mathrm{kcal}$ para la pintada). La pintada atracada digirió mal el grano crudo, de manera que el método de medida de la concentración energética no fue conveniente para esta especie.

Palabras clave: Cajanus cajan - Pollo - Gallina de guinea Valor energético. 